\title{
Proliferation but Not Migration Is Associated with Blood Vessels during Development of the Rostral Migratory Stream
}

\author{
Kai Nie Zoltán Molnár Francis G. Szele \\ Department of Physiology, Anatomy and Genetics, University of Oxford, Oxford, UK
}

\section{Key Words}

Subventricular zone $\cdot$ Telencephalon • Olfactory bulb •

Phosphohistone $3 \cdot$ Blood vessels

\begin{abstract}
Blood vessels play a critical role in regulating neural stem cell proliferation and migration. We show here that blood vessels became progressively aligned in the direction of the rostral migratory stream (RMS) from embryonic day 14 to postnatal day 4. Dividing cells revealed by phosphohistone $\mathrm{H} 3+$ immunoreactivity were statistically closer to isolectin B4+ blood vessels than predicted by chance in the emerging RMS. The close proximity of blood vessels and $\mathrm{H} 3+$ cells was consistent regardless of the age of the RMS and was strikingly similar to the embryonic cerebral cortex. In contrast to the adult RMS, we found no evidence for preferential juxtaposition of migratory doublecortin-positive neuroblasts and vasculature in the neonatal RMS. Our work provides an important framework for understanding the precise mechanism behind regulation of proliferation.
\end{abstract}

Copyright $\odot 2010$ S. Karger AG, Base

\section{Introduction}

Neurogenesis has been well characterized in two highly specialized neural stem cell niches of the adult mammalian brain, the subventricular zone (SVZ) and the sub- granular zone of the hippocampal formation [AlvarezBuylla and Lim, 2004]. SVZ-derived neuroblasts migrate through the rostral migratory stream (RMS) to the olfactory bulb, where they differentiate into inhibitory granule and periglomerular interneurons. While SVZ-derived neuroblasts express neuron-specific markers, they nonetheless retain the capacity for concurrent proliferation and migration [Luskin et al., 1997]. The proliferative capacity in particular is a prominent feature of the emerging RMS during development [Pencea and Luskin, 2003]. With increasing understanding of niche components that regulate neural stem cell behaviour in vivo [Riquelme et al., 2008], it is not at all unreasonable to speculate that a similar niche may exist for proliferative neuroblasts migrating in the embryonic and early postnatal RMS.

It is now recognized that vasculature plays a critical role in neural stem cells. Co-culturing with vascular endothelium stimulates neural stem cell self-renewal and inhibits their differentiation in vitro, mediated by soluble factors from endothelial cells [Shen et al., 2004]. A number of candidate molecules have been put forward including Notch, vascular endothelial growth factor, fibroblast growth factor 2, pigment epithelium-derived factor and brain-derived neurotropic factor [Leventhal et al., 1999; Ramírez-Castillejo et al., 2006; Wada et al., 2006; Kerever et al., 2007]. A specialized vasculature in the adult SVZ in vivo has recently been described [Shen et al., 2008; Tavazoie et al., 2008]. Mitotic glial fibrillary acidic protein-positive (GFAP+) neural stem cells lie in close asso-

\section{KARGER}

() 2010 S. Karger AG, Basel

Fax +41613061234 E-Mail karger@karger.ch www.karger.com
Accessible online at: www.karger.com/dne
Zoltán Molnár or Francis Szele, Department of Physiology, Anatomy and Genetics University of Oxford, Le Gros Clark Building, South Parks Road Oxford OX1 3QX (UK)

Tel. Z.M +44 1865282 664, Tel. F.S. +44 1865282 627, Fax +44 1865272420

E-Mail Zoltan.Molnar@dpag.ox.ac.uk or Francis.Szele@dpag.ox.ac.uk 
ciation to SVZ vascular plexi. The blood brain barrier in the SVZ is more permeable than outside the niche; small molecules like sodium fluorescein access the SVZ directly from blood vessels. Specialized vascular sites that lack astrocyte endfeet and pericytes appear to act as a scaffold for seeding regeneration by neural stem cells following elimination of transit-amplifying cells and neuroblasts with the anti-mitotic drug cytosine- $\beta$-D-arabinofuranoside [Tavazoie et al., 2008]. The laminin receptor $\alpha 6 \beta 1$ integrin mediates adhesion of SVZ neural stem cells to endothelial cells in vitro, and blocking this alters progenitor cell position in relation to blood vessels in vivo [Shen et al., 2008]. This suggests a possible role for 'fractones', slender laminin-containing extravascular basal lamina, in niche regulation [Mercier et al., 2002]. The relationship between proliferation and blood vessels has also been described in the embryonic cerebral cortex, where mitotic cells are closer to blood vessels than predicted by chance [Javaherian and Kriegstein, 2009; Stubbs et al., 2009]; however, their existence in the emerging RMS has not been examined.

It is conceivable that blood vessels have a similar relationship to proliferative cells migrating in the embryonic RMS. It is not known whether the vasculature has the same effect on embryonic neurogenesis in forebrain germinal zones and whether the presumptive vasculature assumes the same architecture during the embryonic to neonatal transition. Many similarities exist between signalling molecules and patterns of division of embryonic and adult neurogenesis [Molnar et al., 2009], suggesting that their neurovascular niches may be similar. A further question at this point is whether blood vessels promote and guide the migration of neuroblasts in the emerging RMS as they apparently do in the adult SVZ [Bovetti et al., 2007; Whitman et al., 2009]. This is likely given the striking parallels between the molecular mechanisms of guidance in vascular and axonal growth [Carmeliet and Tessier-Lavigne, 2005; Weinstein, 2005]. In the peripheral nervous system, artemin expressed on developing vasculature instructs emerging sympathetic axons to follow 'pioneer vessels' as they reach their final targets [Honma et al., 2002]. However, the precise relation between vasculature and neuronal migration is far from conclusively understood. Therefore, it is relevant to examine the influence of the vasculature in vivo on migration as well as proliferation of RMS neuroblasts during embryonic and neonatal stages.

In the present study, we first characterized the changing patterns of vascular architecture in the rostral forebrain through the critical transition between embryonic
Table 1. Numbers of mice used in our experiments

\begin{tabular}{lllll}
\hline Age & IB4 & H3 & H3/Tbr2 & Dcx \\
\hline E14 & 3 & 3 & 3 & - \\
E16 & 3 & 3 & - & - \\
E18 & 3 & 3 & - & - \\
P2 & 3 & 3 & - & - \\
P4 & 3 & 3 & - & 3 \\
\hline
\end{tabular}

IB4 reveals blood vessels, H3 reveals dividing cells, and Dcx reveals migrating neuroblasts. Tbr2 is a transcription factor expressed by intermediate progenitor cells during cortical neurogenesis.

and early postnatal ages. We then examined the spatial relationship of proliferative neuroblasts to blood vessels within the RMS using immunohistochemistry and confocal microscopy. Finally, we looked for a possible role for blood vessels in neuronal migration. Here, we demonstrate that, in vivo, dividing cells are statistically closer to blood vessels than would be predicted by chance. This vasculature appears to undergo extensive reorganization between embryonic and early postnatal ages to take on the architecture of blood vessels aligned in the direction of the RMS.

\section{Materials and Methods}

\section{Animals}

C57-Bl6 mice were obtained from the Departmental Animal Colony at the University of Oxford, UK. All animal procedures were approved by a local Ethical Review Committee and performed under a UK Home Office licence (Scientific Procedures Act, 1986). Embryonic day 0 (E0) is defined as the day of plug detection. Time-mated pregnant mice were killed by cervical dislocation, and then the embryos were obtained by Caesarean section. The heads were removed and then post-fixed overnight in $4 \%$ paraformaldehyde (TAAB, Reading, UK) in $0.1 \mathrm{M} \mathrm{PBS} \mathrm{(pH} \mathrm{7.4)} \mathrm{at}$ $4^{\circ} \mathrm{C}$. Postnatal animals were killed by a lethal injection of pentobarbitone (Euthatal $150 \mathrm{mg} / \mathrm{kg}$ intraperitoneally; Merial Animal Health Ltd., Harlow, UK) and then fixed by transcardial infusion of $4 \%$ paraformaldehyde. Fixed heads were stored in $0.1 \mathrm{M} \mathrm{PBS}$ plus $0.05 \%$ sodium azide at $4^{\circ} \mathrm{C}$. Table 1 summarizes the number of mice used in the study.

\section{Immunohistochemistry}

Fixed whole brains were removed from the skulls under a Nikon SMZ645 dissecting microscope. Brains were embedded in $4 \%$ agarose and cut into $40-\mu \mathrm{m}$ sagittal sections in $0.1 \mathrm{M}$ PBS using a Leica VT1000S vibrotome. Sections were washed 3 times for $15 \mathrm{~min}$ each time in $0.05 \mathrm{M}$ Tris-buffered saline (TBS) on a rocker, 
blocked in $10 \%$ normal goat serum or $10 \%$ normal donkey serum in TBS plus $0.1 \%$ Triton-X for $1 \mathrm{~h}$ at room temperature and then incubated with primary antibodies diluted in $1 \%$ normal goat serum/normal donkey serum and TBS overnight at $4^{\circ} \mathrm{C}$. Sections were again washed 3 times for $15 \mathrm{~min}$ each in TBS and incubated with appropriate secondary antibodies and fluorescein Griffonia simplifolia (Bandeiraea) isolectin B4 (IB4; 1:200; Vector Laboratories), which reveals blood vessels by binding to carbohydrate residues on their luminal surface [Alroy et al., 1987], for $1 \mathrm{~h}$. Sections were counterstained with DAPI (1:1,000; Invitrogen) for 10 min following three 15-min washes in TBS, mounted on glass slides and then coverslipped with Slowfade ${ }^{\circledR}$ Antifade (Invitrogen) for confocal imaging.

The primary antibodies used were anti-phosphohistone $\mathrm{H} 3$, rabbit IgG (1:500; Upstate) or mouse IgG (1:2,000; Abcam); antiT-box brain 2 (Tbr2), rabbit IgG (1:2,000; a gift from Robert Hevner), and anti-doublecortin (Dcx), goat IgG (1:500; Santa Cruz Biotechnology). The secondary antibodies used were goat anti-mouse Cy5 (1:500; Jackson Immunoresearch); goat anti-rabbit Alexa546 (1:500; Molecular Probes), and donkey anti-goat Alexa568 (1:500; Invitrogen).

\section{Image Acquisition}

Mounted sections were imaged with a Leica DMR upright microscope with an attached Leica DFC500 camera, and fluorescent micrographs were acquired using Leica FireCam software. Individual RGB channels were combined using Adobe Photoshop CS3 software. For quantitative analysis of $\mathrm{H} 3$ immunohistochemical data, Z stacks (optical thickness 2.4 or $4.8 \mu \mathrm{m}$ ) were collected using a Zeiss 710 confocal microscope at $\times 20$ or $\times 40$ magnification. The entire RMS was imaged sequentially following its course through the rostral forebrain and was reconstructed from the montage of collapsed Z stacks. Z stacks were analysed individually, but data for one individual age were pooled.

\section{Image Analysis and Quantification}

All image analysis and quantification was performed using ImageJ software (NIH, Bethesda, Md., USA). We examined whether dividing cells are statistically closer to blood vessels than chance under the framework of the 'sphere of influence' model (fig. 1; adapted from Stubbs et al. [2009]). The model imagines a blood vessel as a line of infinitely small dots joined together, and there is a sphere of influence surrounding each dot, within which neurons fall under the influence of this particular blood vessel [Stubbs et al., 2009]. The most important parameter for a sphere is its radius ( $\mathrm{R})$, which represents the maximum distance from a blood vessel that a cell can survive depending on that vessel alone. $\mathrm{R}$ is calculated by halving the average distances between adjacent blood vessels and is obtained for different brain regions and for different ages. Through integration, we can calculate a theoretic average distance (theoretical $r$ ) a cell can expect to be from the centre of the sphere of influence. This value serves as a yardstick against which the actual observed average distance (observed $r$ ) of a dividing cell from the most adjacent blood vessel was compared. Therefore, we can quantitatively assess whether a mitotic cell is closer to a blood vessel than a quiescent cell can be expected to be. The forebrain region was optically imaged through the entirety of a brain slice $(40 \mu \mathrm{m})$. Areas at the upper and lower extremes of each $40-\mu \mathrm{m}$-brain slice were likely to be under the influence of nearby blood vessels that were physically cut away from

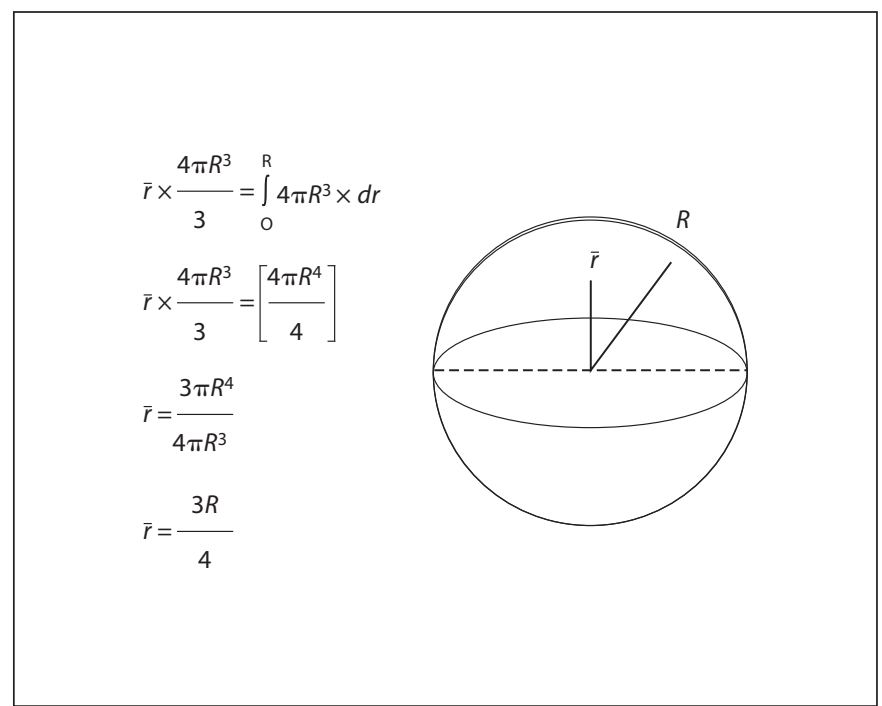

Fig. 1. The 'sphere of influence' model (adapted from Stubbs et al. [2009]). A simple analogy can be drawn between this model and a gumball machine. Imagine a spherical gumball machine completely filled with balls. An imaginary line is drawn between the centre of the gumball machine and the centre of each and every ball. All individual distances are added up and then divided by the total number of balls to give an average distance. The reasoning behind this model is the same except that it assumes all the balls are infinitely small. A straightforward mathematical function is performed by integration to sum up the infinite number of imaginary lines. In the end, an average distance from the centre of the sphere is calculated.

the actual brain slice. Therefore, only the innermost part of the brain slice (at least $\mathrm{R} \mu \mathrm{m}$ from the superior or inferior surface) were analysed to eliminate such possibilities. The RMS at E16, E18 and postnatal day 4 (P4) was analysed to determine the extent of vascular influence on neuroblast proliferation during embryonic to postnatal transition, with E14 cortex, a region of well-documented embryonic neurogenesis, as control.

\section{Results}

\section{Changing Patterns of Vascular Organization in the Rostral Forebrain}

We examined the pattern of vascular development in the rostral forebrain at different embryonic and early postnatal ages. Blood vessels were revealed with fluorescein Griffonia simplifolia IB4 [Stubbs et al., 2009]. Vascular architecture in the rostral forebrain (fig. 2E-O) followed a pattern of development distinct from that in the cortex (fig. 2A-D). At E14, blood vessels in the rostral forebrain had no apparent organization except for simple 
E14

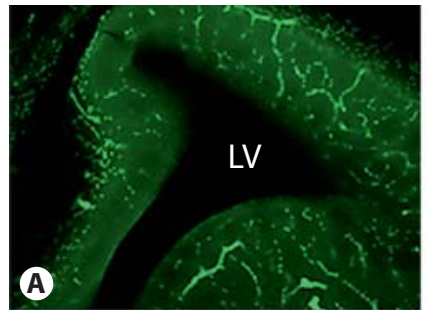

Cortex
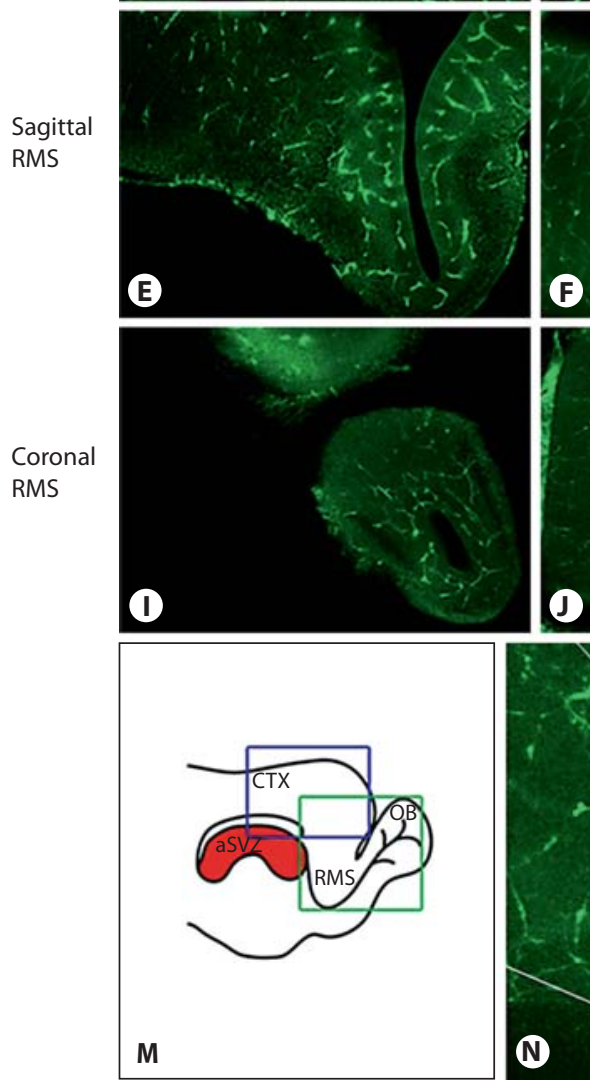

Fig. 2. Development of vascular architecture, as revealed with IB4, in the cortex (A-D) and rostral forebrain region (E-O) at different ages from E14 to P4. The vasculature of the rostral forebrain region has no or little apparent organization at E14 and E16. However, blood vessels appear to align in the direction of the RMS at $\mathrm{P} 2$ and $\mathrm{P} 4$, suggesting extensive remodelling late in development. A, B, E, F Scale bar $=200 \mu \mathrm{m}$. C, D, G, H Scale bar $=500 \mu \mathrm{m}$.

loops of vascular plexi surrounding the rostral extension of the lateral ventricle. Similar vascular loops featured prominently in other brain regions at the same age. At E16, tangentially orientated blood vessels started to appear in the presumptive RMS region. On reaching postnatal ages, blood vessels became progressively more aligned in the longitudinal direction of the RMS and parallel to each other, suggesting that extensive vascular re-
P2
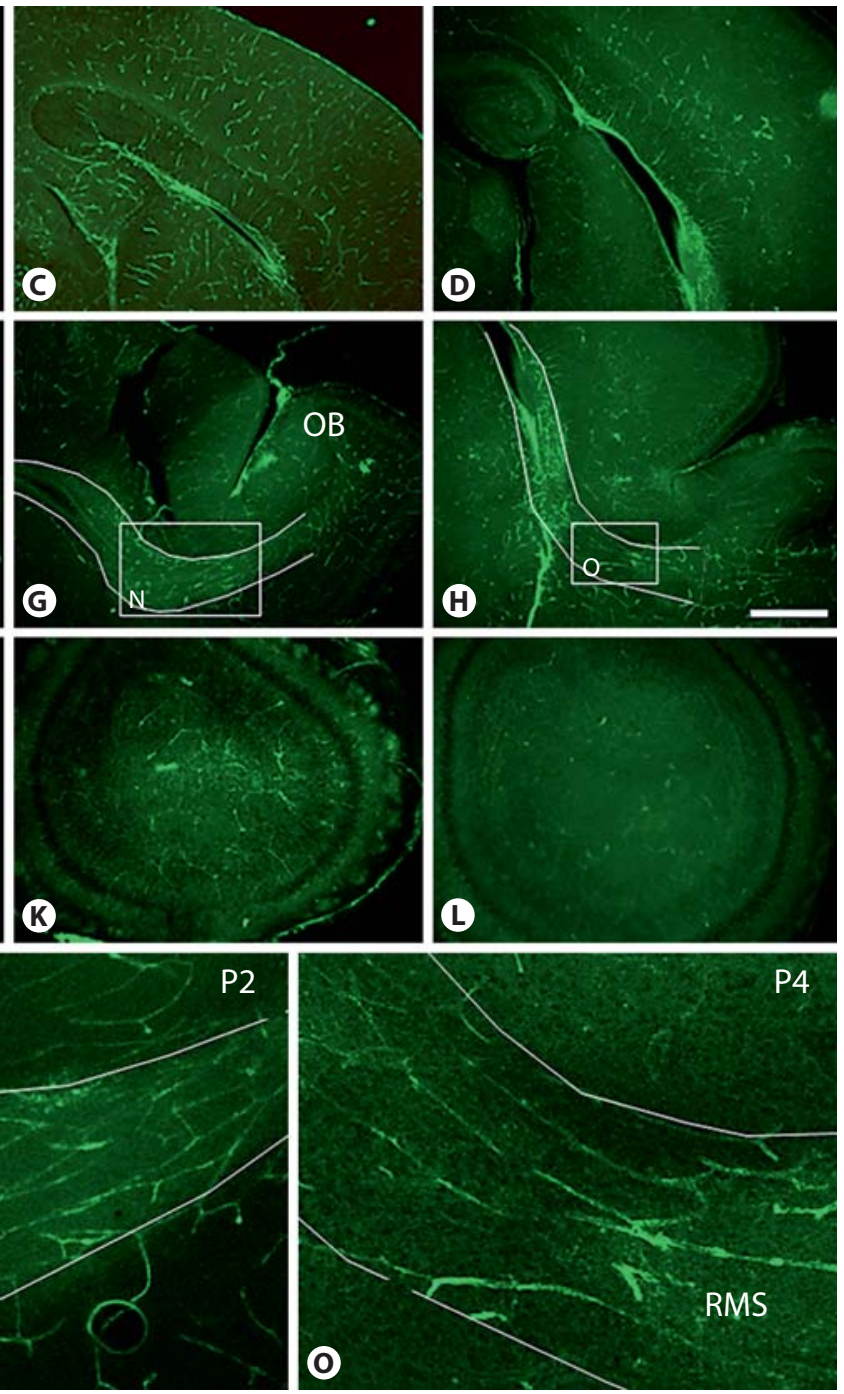

M Schematic drawing of a mouse brain in a sagittal plane. The blue box represents the brain area shown in $\mathbf{A}-\mathbf{D}$ and the green box represents that in $\mathbf{E}, \mathbf{F}$. Insets in $\mathbf{G}$ and $\mathbf{H}$ are shown at higher magnification in $\mathbf{N}$ and $\mathbf{O}$. The RMS as it runs rostral to reach olfactory bulb is outlined in $\mathbf{G}, \mathbf{H}, \mathbf{N}$ and $\mathbf{O}$. CTX = Cortex; $\mathrm{LV}=$ lateral ventricle; $\mathrm{OB}=$ olfactory bulb.

modelling takes place late in development (fig. 2G, H, N, O). This vascular architecture was markedly different from that in both the embryonic cortex (fig. 2A-D), which is best described as looping vascular plexi linked by bridging branches, and at postnatal ages, which is best described as a homogeneous vascular structure with numerous small parenchymal vessels [Bovetti et al., 2007]. 


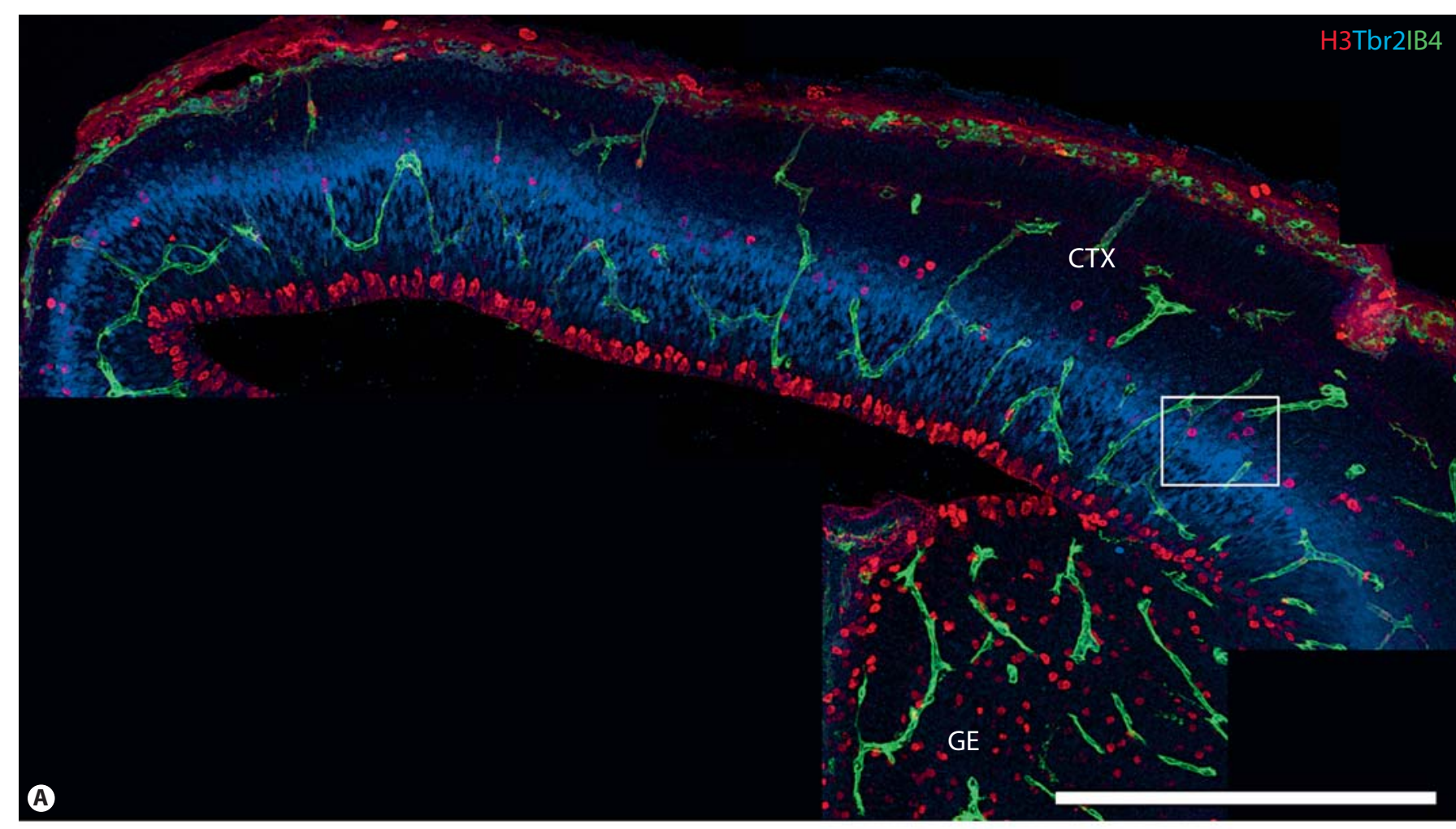

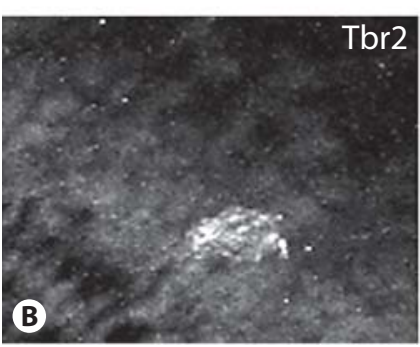

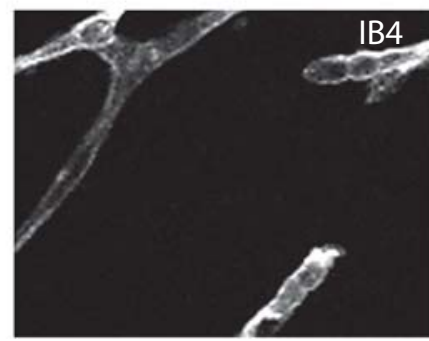

Fig. 3. Embryonic cell proliferation occurs near blood vessels in the cortex. A Confocal optical stacks reveal the overall spatial organization of mitotic intermediate progenitor cells $(\mathrm{H} 3+\mathrm{Tbr} 2+)$ in relation to blood vessels (IB4+) in the embryonic SVZ. As expected, there are no $\mathrm{TB} 2+$ progenitors in the ganglionic eminence

\section{Dividing Cells Lie Close to Blood Vessels}

A vascular niche in embryonic cerebral cortex neurogenic compartments has recently been reported [Javaherian and Kriegstein, 2009; Stubbs et al., 2009]. We examined the spatial relationship between mitotic cells and blood vessels in the embryonic cerebral cortex SVZ before applying the same method to investigate the early postnatal RMS. It has to be emphasized that the embryonic cortical SVZ is not to be confused with the postnatal and adult SVZ, which gives rise to neuroblasts migrating in the RMS to the olfactory bulb [for a review, see Molnar
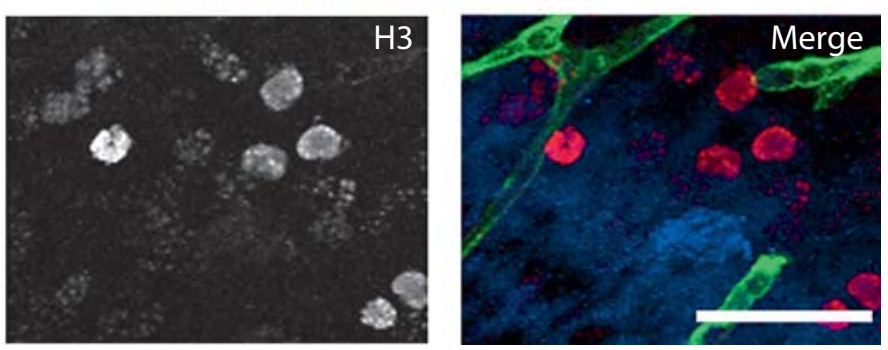

(GE). Scale bar $=400 \mu \mathrm{m}$. CTX $=$ Cerebral cortex. B Higher magnification of the area indicated by the rectangle in $\mathbf{A}$, showing individual channels. They illustrate examples of dividing intermediate progenitor cells that are in close proximity to the cortical vascular plexi. Scale bar $=50 \mu \mathrm{m}$.

et al., 2009]. Dividing cells were revealed with the M phase marker phosphohistone $\mathrm{H} 3$ and blood vessels with IB4 (fig. 3). Embryonic cortical SVZ cells, also known as intermediate progenitor cells, express the transcription factor Tbr2. We examined cells immunoreactive for both $\mathrm{H} 3$ and Tbr2 to confirm that dividing cells are of a neuronal rather than glial lineage. We confirmed that dividing cells indeed lie in close proximity to the looping vascular plexi in the embryonic cortical SVZ (fig. 3A, B), as previously demonstrated by Javaherian and Kriegstein [2009] and Stubbs et al. [2009]. 

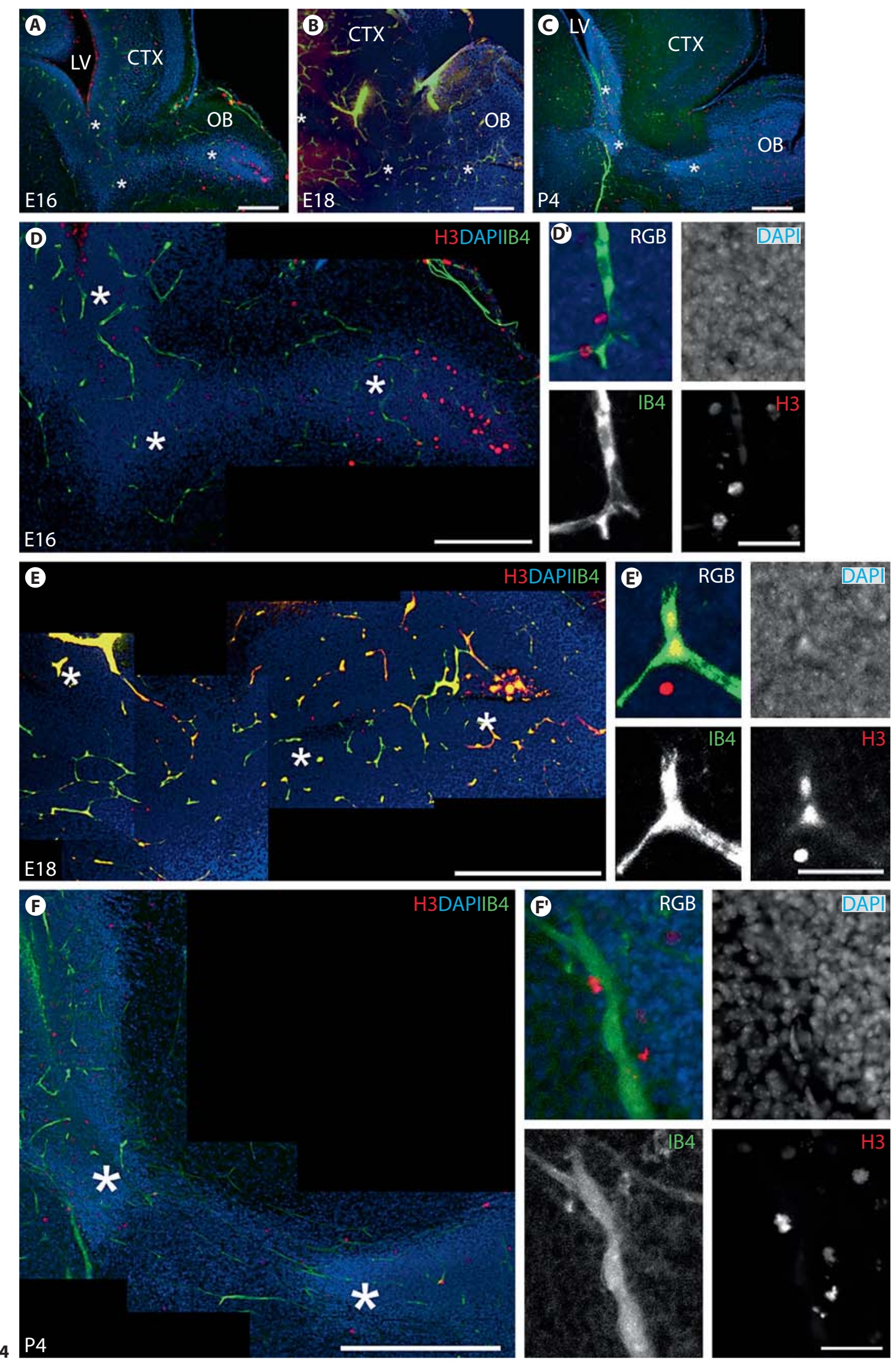


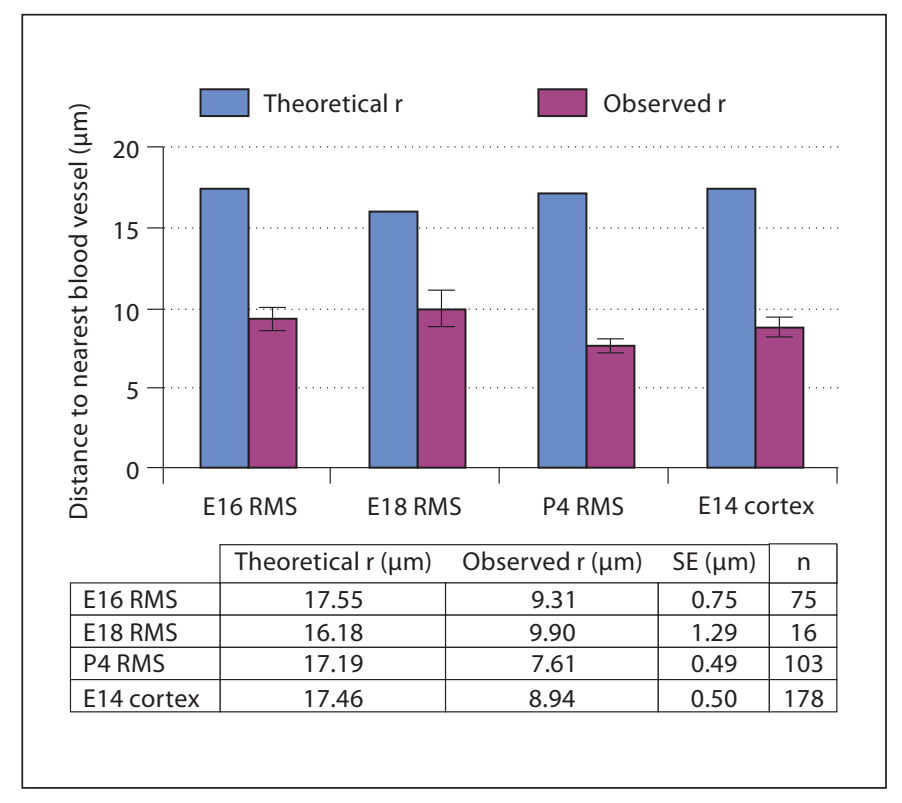

Fig. 5. Quantification of the relationship between mitotic cells and blood vessels. The graph compares the average distance of a mitotic cell from a blood vessel (observed r; red bars) to a theoretical average distance derived from the model for any cells within the sphere of influence (theoretical r; blue bars). Analysis was performed for E16, E18 and P4 RMS, with E14 cortex used as an internal control. Error bars indicate standard errors (SE). The observed $r$ was significantly smaller than the theoretical $r$ in all cases ( $p<0.0005$, one-sample Student's t test). Therefore, mitotic cells were closer to blood vessels than predicted by chance. Furthermore, there was no significant difference in observed $r$ between the RMS of different ages and compared to E14 cortex ( $\mathrm{p}=$ 0.1832, one-way ANOVA).

Fig. 4. Proliferative cells in the RMS lie close to blood vessels at embryonic and early postnatal ages. A-C Low-power fluorescent photomicrographs of the rostral forebrain region at E16, E18 and $\mathrm{P} 4$ reveal the morphological development of the RMS in relation to other forebrain structures. A, B Scale bars $=200 \mu \mathrm{m}$. C Scale bar $=500 \mu \mathrm{m}$. CTX = Cortex $\mathrm{LV}=$ lateral ventricle; $\mathrm{OB}=$ olfactory bulb. Asterisks mark the corresponding RMS at E16, E18 and P4 shown in D-F, respectively. D-F Confocal stacks (optical thickness 2.4 or $4.8 \mu \mathrm{m}$ ) of E16, E18 and P4 RMS, respectively. At E16 and E18, mitotic cells ( $\mathrm{H} 3+)$ are located in close association with blood vessels (IB4+) in the RMS despite vascular plexi at these ages showing no apparent organization with respect to the tangential course of the RMS (densely stained with DAPI). At P4, blood vessels align longitudinally along the direction of the RMS, and mitotic cells, as expected, are intimately associated with blood vessels. D Scale bar $=200 \mu \mathrm{m}$. E Scale bar $=300 \mu \mathrm{m}$. F Scale bar $=400 \mu \mathrm{m}$. D'-F' High-magnification photomicrographs highlighting the close relationship between mitotic cells and blood vessels. Scale bars $=30 \mu \mathrm{m}$.

Embryonic RMS and the Vascular Niche
We then examined the relationship between proliferative H3-immunoreactive cells and blood vessels in the embryonic and early postnatal RMS (fig. 4). Previous studies have convincingly demonstrated that the highly proliferative intermediate progenitor cell population residing within the embryonic RMS is neurogenic [Pencea and Luskin, 2003]. An intimate relationship was strikingly apparent at all ages examined (fig. 4A-C). Dividing cells were closely associated with blood vessels in the RMS at E16 (fig. 4D), E18 (fig. 4E) and P4 (fig. 4F). This relationship seems to be consistent despite the changing vascular architecture, with blood vessels becoming aligned to the direction of the RMS between E16 and P4.

To quantify the spatial relationship between dividing cells and blood vessels, we first calculated the theoretical average distance (theoretical r) a cell would be from the nearest blood vessel as predicted by the 'sphere of influence' model (fig. 1). The theoretical $r$ values for E16, E18 and P4 RMS and E14 cortex were 17.5, 16.5, 17.2 and 17.5 $\mu \mathrm{m}$, respectively (fig. 5). The observed average distance (observed r) of mitotic cells to the nearest blood vessel was significantly smaller than the respective theoretical average distance in all cases (fig. 5). The observed $r$ values for E16, E18 and P4 RMS and E14 cortex were $9.31 \pm 0.75$, $9.90 \pm 1.29,7.61 \pm 0.49$ and $8.94 \pm 0.50 \mu \mathrm{m}$, respectively. For example, a mitotic cell is $47.0 \pm 4.3 \%$ closer to a blood vessel in E16 RMS, $38.8 \pm 8.0 \%$ closer in E18 RMS, $55.7 \pm 2.9 \%$ closer in P4 RMS and $48.8 \pm 2.9 \%$ closer in E14 cortex ( $p<0.0005$ in all cases, one-sample Student's t-test). Therefore, dividing cells in the RMS were closer to blood vessels than predicted by chance. Furthermore, the observed $r$ values were remarkably consistent within the RMS at different ages (E16, E18 and P4) and compared to E14 cortex; differences between them were not statistically significant $(\mathrm{p}=0.1832$, one-way ANOVA). This suggests a common mechanism via which the relationship between the vasculature and mitotic cells is maintained throughout development independent of location and age.

\section{A Possible Role for Blood Vessels in Guiding Migrating Neurons}

We examined the relationship between neuroblasts migrating in the RMS and blood vessels. We hypothesized that blood vessels provide guidance to migratory neuroblasts similar to the mechanism that has been suggested in adults [Whitman et al., 2009]. The cell bodies and leading processes (slender neurites) of migratory neuroblasts were revealed with antibodies against Dcx, a microtubule-associated protein and cell migration 

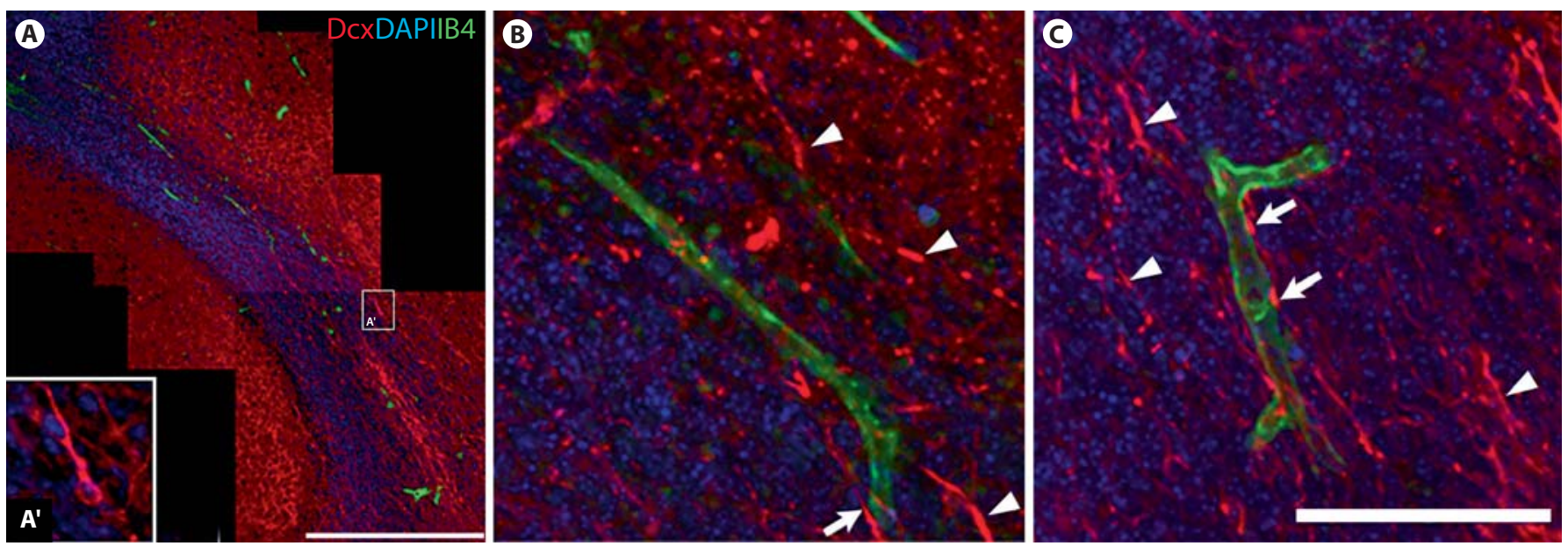

Fig. 6. Relationship between migratory neuroblasts (Dcx+) and blood vessels (IB4+) in P4 RMS. A Montage of confocal images taken in a single optical plane gives an overview of the morphological structure of the RMS. Note that most postmitotic neurons outside the RMS retain Dcx immunofluorescence at this age. $\mathbf{A}^{\prime}$ Example of Dcx+ neuroblast shown at higher magnification. Scale bar $=300 \mu \mathrm{m}$. B, C High-power confocal photomicrographs show a few examples of Dcx + neuroblasts intertwining with blood vessels in the RMS (arrows). However, there is no sign of an intimate association with blood vessels for the vast majority of Dcx+ neuroblasts (arrowheads) migrating in the P4 RMS. This therefore suggests that neuroblasts do not preferentially migrate along blood vessels. Scale bar $=100 \mu \mathrm{m}$. marker [Francis et al., 1999], and neuronal somata were also visualized with the nuclear marker DAPI. We found no evidence to suggest that neuroblasts preferentially migrate along blood vessels in P4 RMS (fig. 6A-C). While we encountered some examples of Dcx+ migratory neuroblasts juxtaposed to blood vessels (fig. 6B, C, arrows), therefore hinting at an intimate association, the vast majority of neuroblast neurites had no or little apparent association with blood vessels (fig. 6B, C, arrowheads).

\section{Discussion}

We show here that the emerging RMS is characterized by proliferation occurring close to blood vessels. We also demonstrate that though blood vessels align with the RMS during late embryonic development, neuroblasts do not migrate preferentially along them.

\section{A Highly Specialized Vascular Architecture in the Embryonic Rostral Forebrain}

We have shown a remarkable level of sophistication in the vascular architecture of the emerging RMS. Blood vessels become progressively aligned to the direction of the RMS during the period of embryonic to postnatal transition (E14-P4), possibly due to extensive vascular pruning late in development. This is apparent by $\mathrm{P} 4$, although the first tangentially orientated blood vessels appear as early as E16. Other studies have demonstrated that in the period following the ages we studied, active migration and proliferation occur in the RMS [Luskin, 1993]. However, the extent to which blood vessels are proximal to and support either proliferation or migration in the postnatal RMS is less clear. CD31+ endothelial tip cells with numerous filopodia were observed in the P3-P7 SVZ, suggesting that endothelial development continues postnatally in that structure [Tavazoie et al., 2008].

Blood vessels may respond to the same environmental cues as neuroblasts of the emerging RMS, consistent with previous observations of extensive overlap in molecular mechanisms of guidance between angiogenesis and axon outgrowth [Carmeliet and Tessier-Lavigne, 2005; Weinstein, 2005]. Alternatively, this may reflect a vascular response to the increasing metabolic demand of an emerging and densely populated RMS through the hypoxiahypoxia-inducible factor-angiogenesis pathway [Pugh and Ratcliffe, 2003]. Either way, this highlights a highly specialized vascular architecture developing alongside the emerging RMS that may well represent a niche permissive for proliferation and migration. 


\section{Dividing Cells Lie Close to Blood Vessels}

We have demonstrated that, in embryonic and early postnatal RMS in vivo, dividing cells are closer to blood vessels than would be predicted by chance. While we recognize that this study shows no direct evidence that the dividing cells will give rise to neurons, previous studies have convincingly shown that mitotic cells in the embryonic RMS are neuroblasts, with expression of neuronspecific markers [Luskin et al., 1997; Pencea and Luskin, 2003]. On the other hand, H3+ cells could possibly be precursors to oligodendrocytes, astrocytes, microglia, endothelial cells or pericytes. Our study points to the possibility of an RMS-specific vascular niche capable of supporting proliferation. The average distance of a dividing cell to the nearest blood vessel is consistent in the RMS at E16, E18 and P4, and in E14 cortex, a region of well-documented embryonic neurogenesis [Stubbs et al., 2009]. The proximity between proliferation and blood vessels is reminiscent of previously described adult neural stem cell niches in the SVZ [Shen et al., 2008; Tavazoie et al., 2008] and subgranular zone [Palmer et al., 2000]. This similarity leads to the important question of the precise mechanism through which such vascular niches maintain the unique neurogenic capacities of these compartments throughout life.

We recognize that the 'sphere of influence' model (fig. 1) [Stubbs et al., 2009], which predicts an average distance for all cells within a sphere of influence, has obvious limitations. The model assumes (1) that cells are dimensionless points and blood vessels are lines joining an infinite number of points and (2) a uniform distribution of cells within a volume of tissue. It therefore represents a greatly simplified view of neuronal organization. It is possible that the neuropil near the vasculature is more sparse, rendering cell density greater. If so, and assuming equal rates of proliferation, one would automatically see more phosphohistone $\mathrm{H} 3+$ cells near the vasculature, because cells are more concentrated there. Nevertheless, the 'sphere of influence' model allowed us to demonstrate the close affinity between vasculature and cellular divisions in a quantitative manner.

We cannot make inferences about molecular mechanisms underlying the intimate physical association between proliferation and vasculature based on our descriptive study; however, there are several avenues worthy of future investigation. Vascular endothelial cells are a known source of soluble neurotropic factors [Leventhal et al., 1999; Ramírez-Castillejo et al., 2006; Wada et al., 2006; Kerever et al., 2007]. Notch and NF- $\kappa$ B are thought to be downstream of neurotropic factors and are impli- cated in neural stem cell self-renewal [Shen et al., 2004] and survival [Wada et al., 2006], respectively. Endothelial cells are known to influence the proliferation and differentiation of transplanted stem cells [Nakagomi et al., 2009]. Enhanced neurogenesis and angiogenesis in the forebrain and in the dentate gyrus of the hippocampus have been reported in transgenic mice overexpressing vascular endothelial growth factor [Udo et al., 2008]. However, the vascular basal lamina also plays an important role in regulating niche behaviour in vivo. The basal lamina traps diffusible tropic signals such as fibroblast growth factor 2 [Kerever et al., 2007]. The laminin receptor $\alpha 6 \beta 1$ integrin tethers neural stem cells to endothelial cells in vitro, and blocking this integrin disrupts adhesion of SVZ neural stem cells to blood vessels in vivo [Shen et al., 2008]. We do not know the extent to which the capacity of the niche to support proliferation can be attributed to diffusible signals or extracellular matrix.

\section{RMS Neuroblasts Do Not Preferentially Migrate along Blood Vessels}

We were surprised to observe no close relationship between Dcx+ migratory neuroblasts and blood vessels in P4 RMS. This is particularly surprising in light of previous reports suggesting a role for blood vessels in guiding adult SVZ neuroblast migration [Bovetti et al., 2007; Whitman et al., 2009]. Sympathetic axons follow 'pioneer vessels' as they migrate outwards to reach their peripheral targets via interaction with artemin expressed on blood vessels [Honma et al., 2002]. Artemin-soaked beads have been shown to encourage neuroblast migration and axonal outgrowth toward themselves when implanted into mouse embryos. Moreover, radial migration of RMS neuroblasts into the granule and periglomerular layers of the olfactory bulb, visualized using in vivo time-lapse imaging, is intimately associated with blood vessels [Bovetti et al., 2007]. However, we cannot exclude the possibility that a subpopulation of RMS neuroblasts do preferentiallymigrate alongblood vessels based on our preliminary finding from immunohistochemical staining for Dcx alone.

\section{Clinical Implications}

An understanding of the precise mechanisms of niche regulation of the proliferation and migration of neuron progenitors has widespread clinical implications. Postmortem examination of patient brains revealed elevated SVZ neurogenesis following ischaemic insults such as stroke, which suggests a self-reparative response to injury [Macas et al., 2006]. Increased SVZ neuronal proliferation 
and stem cell markers have also been reported in multiple sclerosis and Huntington's disease, although their physiological significance is controversial [Curtis et al., 2003; Nait-Oumesmar et al., 2007]. While it may be the case that neural stem cells retain a regenerative capacity to react to injury, any treatment based on this will nonetheless remain elusive unless we decipher how neural stem cell niches regulate neurogenesis, migration and integration.

\section{Acknowledgements}

We thank Jamin Deproto for advice on quantification and confocal microscopy. We thank members of the Molnar and Szele laboratories for critical readings of the manuscript. Z.M. was supported by Medical Research Council grant G0700377 and the Biotechnology and Biological Sciences Research Council grant BB/ F003285/1. F.G.S. was supported by NIH grant RO1 NS-42253.

\section{References}

-Alroy J, Goyal V, Skutelsky E (1987): Lectin histochemistry of mammalian endothelium. Histochemistry 86:603-607.

-Alvarez-Buylla A, Lim D (2004): For the long run: maintaining germinal niches in the adult brain. Neuron 41:683-686

-Bovetti S, Hsieh YC, Bovolin P, Perroteau I, Kazunori T, Puche AC (2007): Blood vessels form a scaffold for neuroblast migration in the adult olfactory bulb. J Neurosci 27:59765980 .

Carmeliet P, Tessier-Lavigne M (2005): Common mechanisms of nerve and blood vessel wiring. Nature 436:193-200.

Curtis MA, Penney EB, Pearson AG, van RoonMom WMC, Butterworth NJ, Dragunow M, Connor B, Faull RLM (2003): Increased cell proliferation and neurogenesis in the adult human Huntington's disease brain. Proc Natl Acad Sci USA 100:9023-9027.

-Francis F, Koulakoff A, Boucher D, Chafey P, Schaar B, Vinet MC, Friocourt G, McDonnell N, Reiner O, Kahn A, McConnell SK, Berwald-Netter Y, Denoulet P, Chelly J (1999): Doublecortin is a developmentally regulated, microtubule-associated protein expressed in migrating and differentiating neurons. Neuron 23:247-256.

Honma Y, Araki T, Gianino S, Bruce A, Heuckeroth R, Johnson E, Milbrandt J (2002): Artemin is a vascular-derived neurotropic factor for developing sympathetic neurons. Neuron 35:267-282.

-Javaherian A, Kriegstein A (2009): A stem cell niche for intermediate progenitor cells of the embryonic cortex. Cereb Cortex 19(suppl 1):i70-i77.

- Kerever A, Schnack J, Vellinga D, Ichikawa N, Moon C, Arikawa-Hirasawa E, Efird JT, Mercier F (2007): Novel extracellular matrix structures in the neural stem cell niche capture the neurogenic factor fibroblast growth factor 2 from the extracellular milieu. Stem Cells 25:2146-2157.

Leventhal C, Rafii S, Rafii D, Shahar A, Goldman SA (1999): Endothelial trophic support of neuronal production and recruitment from the adult mammalian subependyma. Mol Cell Neurosci 13:450-464.
Luskin MB (1993): Restricted proliferation and migration of postnatally generated neurons derived from the forebrain subventricular zone. Neuron 11:173-189.

Luskin MB, Zigova T, Soteres BJ, Stewart RR (1997): Neuronal progenitor cells derived from the anterior subventricular zone of the neonatal rat forebrain continue to proliferate in vitro and express a neuronal phenotype. Mol Cell Neurosci 8:351-366.

-Macas J, Nern C, Plate KH, Momma S (2006): Increased generation of neuronal progenitors after ischemic injury in the aged adult human forebrain. J Neurosci 26:1311413119.

Mercier F, Kitasako JT, Hatton GI (2002): Anatomy of the brain neurogenic zones revisited: fractones and the fibroblast/macrophage network. J Comp Neurol 451:170-188.

Molnar Z, Szele FG, Vercelli A (2009): Forebrain Neurogenic Compartments and Cortical Neurogenesis: Comparative Aspects. Trivandrum, Research Signpost.

Nait-Oumesmar B, Picard-Riera N, Kerninon C, Decker L, Seilhean D, Hoglinger GU, Hirsch EC, Reynolds R, Baron-Van Evercooren A (2007): Activation of the subventricular zone in multiple sclerosis: evidence for early glial progenitors. Proc Natl Acad Sci USA 104: 4694-4699.

Nakagomi N, Nakagomi T, Kubo S, Nakano-Doi A, Saino O, Takata M, Yoshikawa H, Stern DM, Matsuyama T, Taguchi A (2009): Endothelial cells support survival, proliferation, and neuronal differentiation of transplanted adult ischemia-induced neural stem/progenitor cells after cerebral infarction. Stem Cells 27:2185-2195

Palmer TD, Willhoite AR, Gage FH (2000): Vascular niche for adult hippocampal neurogenesis. J Comp Neurol 425:479-494.

Pencea V, Luskin MB (2003): Prenatal development of the rodent rostral migratory stream. J Comp Neurol 463:402-418.

Pugh CW, Ratcliffe PJ (2003): Regulation of angiogenesis by hypoxia: role of the HIF system. Nat Med 9:677-684.
-Ramírez-Castillejo C, Sánchez-Sánchez F, Andreu-Agulló C, Ferrón SR, Aroca-Aguilar JD, Sánchez P, Mira H, Escribano J, Fariñas I (2006): Pigment epithelium-derived factor is a niche signal for neural stem cell renewal. Nat Neurosci 9:331-339.

-Riquelme PA, Drapeau E, Doetsch F (2008): Brain micro-ecologies: neural stem cell niches in the adult mammalian brain. Philos Trans R Soc Lond B Biol Sci 363:123-137.

- Shen Q, Goderie SK, Jin L, Karanth N, Sun Y, Abramova N, Vincent P, Pumiglia K, Temple $S$ (2004): Endothelial cells stimulate self-renewal and expand neurogenesis of neural stem cells. Science 304:1338-1340.

- Shen Q, Wang Y, Kokovay E, Lin G, Chuang SM, Goderie SK, Roysam B, Temple S (2008): Adult SVZ stem cells lie in a vascular niche: a quantitative analysis of niche cell-cell interactions. Cell Stem Cell 3:289-300.

-Stubbs D, DeProto J, Nie K, Englund C, Mahmud I, Hevner R, Molnar Z (2009): Neurovascular congruence during cerebral cortical development. Cereb Cortex 19(suppl 1):i32-i41.

- Tavazoie M, Van der Veken L, Silva-Vargas V, Louissaint M, Colonna L, Zaidi B, GarciaVerdugo JM, Doetsch F (2008): A specialized vascular niche for adult neural stem cells. Cell Stem Cell 3:279-288.

-Udo H, Yoshida Y, Kino T, Ohnuki K, Mizunoya W, Mukuda T, Sugiyama H (2008): Enhanced adult neurogenesis and angiogenesis and altered affective behaviors in mice overexpressing vascular endothelial growth factor 120. J Neurosci 28:14522-14536.

Wada T, Haigh JJ, Ema M, Hitoshi S, Chaddah R, Rossant J, Nagy A, van der Kooy D (2006): Vascular endothelial growth factor directly inhibits primitive neural stem cell survival but promotes definitive neural stem cell survival. J Neurosci 26:6803-6812.

Weinstein BM (2005): Vessels and nerves: marching to the same tune. Cell 120:299302 .

Whitman MC, Fan W, Rela L, Rodriguez-Gil DJ, Greer CA (2009): Blood vessels form a migratory scaffold in the rostral migratory stream. J Comp Neurol 516:94-104. 